\title{
Novel Kv7.1 missense mutation Lys422Glu leads to the development of LQT syndrome
}

Maria Karlova $^{1}$, Valeria Rusinova ${ }^{2}$, Denis Abramochkin ${ }^{1}$, Elena Zaklyazminskaya ${ }^{2}$ and Olga Sokolova ${ }^{1}$

${ }^{1}$ Moscow Lomonosov University, United States, ${ }^{2}$ Russian Scientific Center for Surgery named after B. V. Petrovsky, United States

Electrophysiological activity on cellular level can have significant effect on organism level and even minor changes in conductivity in critically important structures can lead to severe pathologies. One of the examples of this critical functional element is a voltage-dependent potassium channel Kv7.1. In cardiomyocytes Kv7.1 alpha-subunit, encoded by the KCNQ1 gene, is expressed together with the auxiliary subunit KCNE1 to form a functionally active channel. It is responsible for the IKs current of the slow repolarization phase. Mutations of the Kv7.1 channel lead to the development of life-threatening cardiac pathologies [1]. Here we have identified the new point mutation in the KCNQ1 gene, associated with the LQTS. The detected genetic variant in the patient was at the position c.1264A > G (NM_000218.2) leading to the replacement of Lys 422 to Glu in a cytoplasmic domain of Kv7.1 channel alpha-subunit which previously has been not described. Asymptomatic QTc prolongation (QTc $480 \mathrm{~ms}$ resting, and up to $587 \mathrm{~ms}$ in orthoprobe) was found in 4 y.o. male proband from consanguineous family of Daghestani origin during routine ECG screening (Figure 1.A). No family history of sudden cardiac death cases or syncope was disclosed. During target screening of the KCNQ1, KCNH2, KCNJ2, KCNE1-2, SCN5A, SCN1B-4B genes a rare genetic variant c.1264A >G (p.Lys422Glu) in the KCNQ1 gene was found. This mutation was qualified as a variant of unknown significance (VUS) according to ACMG2015 criteria. Cascade familial genetic screening revealed this variant in mother and older brother but not in sister. All variant's carriers had QTc prolongation, and non-carrier (father and sister) had normal ECG parameters. The study was performed with written inform consent of full-aged family members. DNA samples were extracted from the venous blood by standard protocol. Target sequencing of the KCNQ1, KCNH2, KCNJ2, KCNE1-2, SCN5A, SCN1B-4B genes was performed on IonTorrent PGM (Thermo Fisher, USA) platform. All genetic variants were confirmed by Sanger sequencing. To evaluate the electrophysiological features of this genetic variant, the c.1264A $>\mathrm{G}$ point mutation has been introduced into the recombinant KCNE1-KCNQ1 fusion protein. This genetic construct consist of KCNQ1 coding sequence introduced in frame immediately after the KCNE1 subunit sequence without stop-codon, so both subunits are covalently bound after protein translation. Affinity tags were added to the C-terminus of construct to facilitate protein detection. The fusion protein is functionally active $[2,3]$. The plasmid was transfected to the Chinese hamster ovary cells (CHO-K1). The integral IKs current was recorded using the standard whole-cell patchclamp. The currents were elicited by square-pulse depolarization to $-60-80 \mathrm{mV}$ from the holding potential of $80 \mathrm{mV}$ with $20 \mathrm{mV}$ increment. The Lys422Glu substitution in the KCNQ1 leads to a decrease of the IKs current density (Figure 1), but not to a change in the current amplitude or kinetics. Immunoblot and fluorescent microscopy were used for protein expression monitoring. No significant decrease of the total KCNE1-KCNQ1 protein expression in the cells, transfected with mutated sequence variant, was reported based on immunoblot (Figure 2, A). Immunostaining confirmed the presence of a target protein in the cell membrane both for wild type and mutant channels via a clear signal along the cell periphery with similar signal intensity for both proteins (Figure 2, B). Based on the results of experiments the Lys422Glu mutation can be considered as having impact on the Kv7.1 channel conductivity than on membrane traffic modulation. The work was supported by RFBR, \# 20-54-15004. The research was carried out using the equipment purchased on account of the Lomonosov MSU Development Program. 



Figure 1. Patch-clamp recording $\mathrm{CHO}$ cells. Comparison of IKs current carried by control and mutant Kv7 potassium channels in transfected $\mathrm{CHO}$ cells.Left panel: original recordings of current induced by square-pulse depolarization from the holding potential of $-80 \mathrm{mV}$ in representative $\mathrm{CHO}$ cells transfected with control gene of Kv7.1 channels (top) and mutant variant of this gene (bottom).Right panel: comparison of IKs mean I-V curves (top) and activation kinetics (bottom) in CHO cells transfected by control $(n=13)$ and mutant $(n=15)$ Kv7.1 gene.* - significant difference between 2 groups, $\mathrm{p}<0.05$, two-way ANOVA with Tukey's post-hoc test.

A

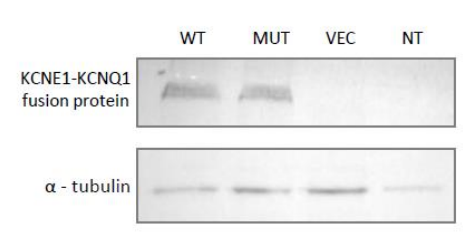

B

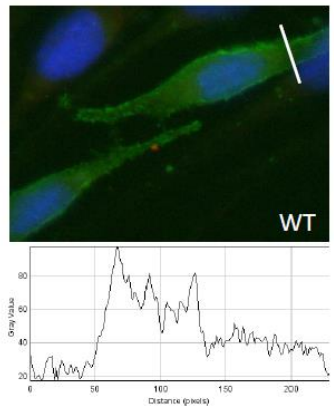



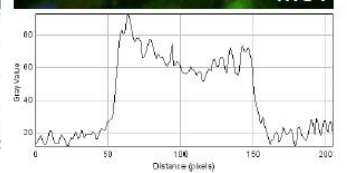

Figure 2. Expression of the recombinant Kv7.1 protein in $\mathrm{CHO}$ eukaryotic cells. A. Immunoblot, $1^{\circ} \mathrm{Ab}$ to $\mathrm{V} 5-$ tag and $\alpha$-tubulin. WT - cells, transfected with control Kv7.1 protein, MUT - cells, transfected with Kv7.1 protein with Lys422Glu mutation, VEC - cells, transfected with an empty vector, NT - non transfected control cells. B. Immunofluorescent staining of $\mathrm{CHO}$ cells expressing the recombinant $\mathrm{Kv} 7.1$ protein: wild-type (top left) and mutant (top right). $1^{\circ} \mathrm{Ab}$ to $\mathrm{V} 5$-tag, $2^{\circ} \mathrm{Ab}$ conjugated to alexa-488 dye (green channel). Bottom panels - intensity of green signal across the cell (along the white lines in the images in the top row) with the two peaks corresponding to cell plasma membrane.

\section{References}

1. Giudicessi JR, Wilde AAM, Ackerman MJ. Trends Cardiovasc Med. 2018;28(7):453-464. doi:10.1016/j.tcm.2018.03.003

2. Wang W, Xia J, Kass RS. J Biol Chem. 1998;273(51):34069-34074. doi:10.1074/jbc.273.51.34069

3. Karlova MG, Voskoboynikova N, Gluhov GS, et al. Chem Phys Lipids. 2019; 219:50-57. doi:10.1016/j.chemphyslip.2019.01.013 